\title{
RöFo 2020: Crowd Review - eine neue Begutachtungsoption
}

Die Qualität wissenschaftlicher Arbeiten wird traditionell durch das Peer Review gesichert [1, 2]. Bei der RöFo setzen wir auf das Double-Blind-Review, bei dem zwei bis drei Gutachter die Arbeit für die Verfasser anonymisiert bewerten.

Das Peer Review-Verfahren nimmt viel Zeit in Anspruch, bis ein abschließendes Votum vorliegt [1]. Zudem nimmt mit der steigenden Zahl von Arbeiten, die begutachtet werden müssen, die Belastung der Peers kontinuierlich zu. Diversifizierung und Spezifizierung in den Fachgebieten lässt andererseits den Kreis der potenziellen Gutachter, die eine Arbeit in ihrer Gesamtheit beurteilen können, kleiner werden - ein Problem, welches uns dazu auffordert, nach neuen Modellen und Lösungsansätzen für die Qualitätssicherung wissenschaftlicher Publikationen zu suchen.

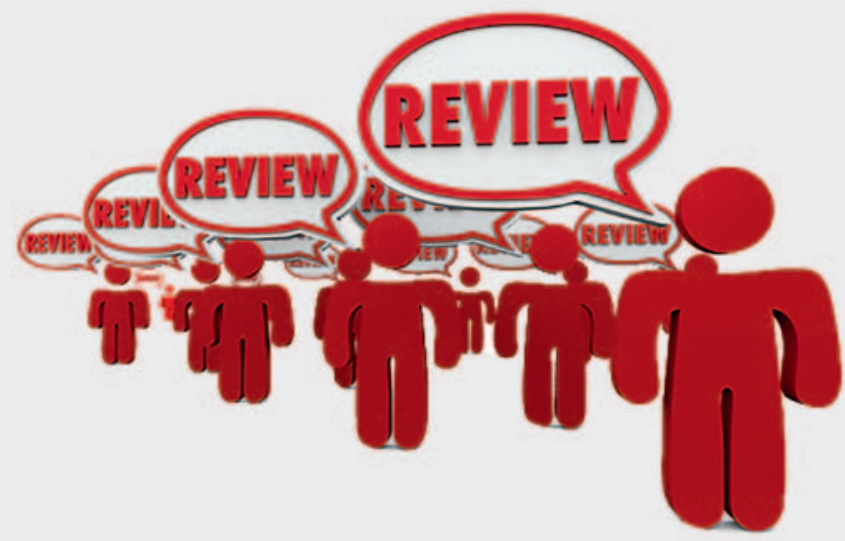

Symbolbild. Quelle: @iQoncept-stock.adobe.com

\section{Crowd Reviewing - viele kleine Gutachter-Beiträge für ein großes Ganzes}

Ab Oktober 2019 führen wir bei der RöFo „intelligentes Crowd Reviewing“ (CR) ein, bei dem eine Gruppe von Experten gemeinsam und anonym eine Arbeit begutachtet - ein innovativer, effizienter Ansatz für die Bewertung wissenschaftlicher Manuskripte [3, 4]. Unser Hauptziel beim CR ist es, verschiedene Verbesserungsvorschläge von mehr als den herkömmlich eingesetzten zwei bis drei Gutachtern zu erhalten, eine lebendige wissenschaftliche Diskussion zu initiieren und eine Entscheidung in vergleichsweiser kurzer Zeit zu erhalten.

Die Gutachter der „Crowd“ kommentieren das Manuskript anonym in einem geschlossenen System. Hierbei kann ein Gutachter ggf. auf Einzelaspekte der Arbeit fokussieren. Die Summe aller Teile führt schließlich zur Gesamtbeurteilung der wissenschaftlichen Arbeit. Da die „Crowd“-Gutachter parallel und nicht seriell an der Begutachtung arbeiten, kann der Ablauf beschleunigt werden und schneller ein abschließendes Votum zu dem Manuskript erzielt werden. Bestätigt hat dies eine erste Evaluation durch die Zeitschrift „Synlett“, die das CR bereits seit einiger Zeit anbietet [3, 4].

Ein weiterer Vorteil des Crowd Review gegenüber dem „klassischen“ Peer Review ist, dass die Gutachter in einen Gedankenaustausch über ihre Kommentare treten können, wobei die Anonymität auch der Gutachter untereinander gewahrt bleibt. 


\section{Zwei Arbeitsgemeinschaften der DRG unterstützen das Projekt}

Als Pilotprojekt und um entsprechende Erfahrungen in unserer radiologischen Community zu sammeln, wollen wir das CR in den Bereichen Abdominaldiagnostik und Uroradiologie anbieten. Herr Professor Hamm hat das CR den beiden Arbeitsgemeinschaften der DRG auf ihren Mitgliederversammlungen anlässlich des Deutschen Röntgenkongresses in Leipzig vorgestellt. Wir danken den Arbeitsgruppen in der DRG, der „AG Gastrointestinal-/Abdominaldiagnostik“ unter dem Vorsitz von Prof. Dr. med. Lars Grenacher und der „AG Uroradiologie und Urogenitaldiagnostik“ unter dem Vorsitz von PD Dr. med. Lars Schimmöller, dass sie ihre Unterstützung für das Projekt spontan zugesagt und aus ihren Reihen mehrere Experten für die „Crowd“ benannt haben.

Als Crowd Review Editor haben wir Herrn PD Dr. Dominik Geisel, Klinik für Radiologie, Charité - Universitätsmedizin Berlin, gewinnen können. Er wird in dieser Funktion die Review-Prozesse betreuen.

Wir freuen uns, Ihnen als Autorinnen und Autoren diesen neuen Ansatz zunächst für die oben genannten Bereiche als zusätzliche (und freiwillige) Begutachtungs-Option bei der Einreichung Ihrer Beiträge anbieten zu können. Sie können somit als Autorin bzw. Autor nun wählen, ob Ihre Arbeit im klassischen Peer Review Prozess oder durch ein Crowd Review beurteilt werden soll, auf Wunsch ist auch eine Kombination aus Peer Review und Crowd Review möglich.

Im Namen der Herausgeber der RöFo und des Thieme Verlags danken wir allen Beteiligten herzlich für ihr Mitwirken an diesem ambitionierten Projekt! Mit Ihnen sind wir gespannt auf diese neue Form der Begutachtung und werden sie kritisch begleiten und über erste Ergebnisse und Eindrücke zeitnah in RöFo berichten.

Wir freuen uns auf Ihre Manuskripte für RöFo!

Gerhard Adam, Bernd Hamm, Walter Heindel, Konstantin Nikolaou (Schriftleiter der RöFo)

Dominik Geisel (Crowd Review Editor, RöFo)

\section{Literatur}

[1] Schmitz ]. Handbuch CoScience/Peer Review. https://handbuch.tib.eu/w/Handbuch_CoScience/ Peer_Review. Last Access 29.07.2019

[2] Johnson R, Watkinson A, Mabe M. 50 1968-2018 Celebrating the $50^{\text {th }}$ Anniversary of STM. $5^{\text {th }}$ ed. International Association of Scientific, Technical and Medical Publishers; 2018

[3] List B. Crowd-based peer review can be good and fast. Nature 2017; 546: 9. doi:10.1038/546009a

[4] Nguyen TM. The cate for crowd peer review. c\&en Chemical\&Engeneering News 2018; 96 